\title{
ISMP Adverse Drug Reactions
}

\section{Herpes Zoster Caused by Zoster Vaccine in an Immunocompetent Patient}

\section{Restless Leg Syndrome Due to the Combination of Venlafaxine and Quetiapine}

\section{SSRIs and the Risk of Upper Gastrointestinal Bleeding}

\section{Minocycline-Induced Cutaneous Polyarteritis Nodosa}

\author{
Michael A. Mancano, PharmD"
}

\begin{abstract}
The purpose of this feature is to heighten awareness of specific adverse drug reactions (ADRs), discuss methods of prevention, and promote reporting of ADRs to the US Food and Drug Administration's (FDA's) MEDWATCH program (800-FDA-1088). If you have reported an interesting, preventable ADR to MEDWATCH, please consider sharing the account with our readers. Write to Dr. Mancano at ISMP, 200 Lakeside Drive, Suite 200, Horsham, PA 19044 (phone: 215-7074936; e-mail: mmancano@temple.edu). Your report will be published anonymously unless otherwise requested. This feature is provided by the Institute for Safe Medication Practices (ISMP) in cooperation with the FDA's MedWATch program and Temple University School of Pharmacy. ISMP is an FDA MEDWATCH partner.
\end{abstract}

\section{HERPES ZOSTER CAUSED BY ZOSTER VACCINE IN AN IMMUNOCOMPETENT PATIENT}

A 68-year-old Hispanic female received zoster vaccine (Zostavax) in her left deltoid region in March 2012. Zostavax contains the Oka/Merck strain of live, attenuated varicella zoster virus (VZV). This patient was monitored as part of a large herpes zoster study in progress at Kaiser Permanente Southern California (KPSC). In December 2012, the patient visited her physician with a 3-day history of rash, mild but annoying pain, and a burning sensation on her left middle chest and middle back as well as under her left arm. She reported no prodromal pain. Her medication history contained no medications that were immunosuppressive, and her medical history contained no immunosuppressive conditions. She reported an uncertain history of varicella. Her physician diagnosed her with herpes zoster infection and initiated acyclovir $800 \mathrm{mg} 5$ times daily for 10 days and acetaminophen for pain. The patient's pain and rash resolved within 10 days and the patient experienced no complications.

Specimens from this patient were submitted to the National Varicella Zoster Vaccine (VZV) Laboratory and Centers for Disease Control and Prevention for VZV strain identification. The specimens submitted for this patient contained isolates that were identified as strains originating from Oka vaccine via FRET real-time polymerase chain reaction, which can discriminate vaccine from wild-type VZV. The fixed ORF62 SNP at positions 106262 and 107252 definitively identified this isolate as Oka vaccine. Therefore

\footnotetext{
${ }^{*}$ Chair and Clinical Professor, Department of Pharmacy Practice, Temple University School of Pharmacy, Philadelphia, Pennsylvania; Clinical Advisor, Institute for Safe Medication Practices, Horsham, Pennsylvania
} 
a single vaccine-associated strain was responsible for this patient's case of herpes zoster.

Almost all zoster vaccine recipients have had primary wild-type VZV infection earlier in life, with establishment of latency in dorsal root ganglia and a robust immune response that protects against a second episode of varicella. Similar to wild-type VZV, vaccine Oka (vOka) is capable of establishing latency in neurons following varicella vaccination. This phenomenon has been well documented in both healthy and immunocompromised individuals, although herpes zoster due to vOka appears to be uncommon. The case the authors presented provides evidence indicating that vOka found in zoster vaccine can establish latency and reactivate to cause clinical zoster. Zostavax contains the same live-attenuated Oka seed stock of VZV that is used for the monovalent varicella vaccine used in the United States (Varivax), but the infectious dose is at least 14 times higher.

The authors point out that a limitation of their findings is that they were unable to confirm a past primary infection with varicella either by history or presence of VZV-specific antibodies prior to receipt of zoster vaccine. The authors surmise that due to the fact that the patient was 68 years old, was born in California, and had spent her entire life in the United States, she was likely exposed to VZV for many decades during which varicella was circulating widely prior to routine varicella vaccination for children. Rates of VZV infection in this cohort of US residents are virtually $100 \%$. The patient also reported that she lived with a large number of siblings during childhood and recalled that several of them had contracted varicella, further reducing the likelihood that she was never infected with wild-type VZV. The authors point out that this case likely represents Oka strain superinfection in the face of prior wild-type immunity. Although highly unlikely, the authors cannot rule out the possibility of a rare VZV-naïve 68-year-old person, with zoster vaccine serving as her primary infection.

To put this case into context, the authors point out that to date more than 16 million doses of zoster vaccine have been distributed by Merck, yet this is the only reported case of Oka strain herpes zoster reported in a patient after receiving zoster vaccine. When combining the findings of the Shingles Prevention Study (SPS) and the author's study, there have been 634 zoster vaccine recipients who developed herpes zoster and were tested for VZV and for VZV strain type, with just 1 case $(0.16 \%)$ of vOka VZV detected. The overwhelming majority of herpes zoster cases occurring among vaccine recipients are due to the limited ability of zoster vaccine to prevent reactivation of wild-type virus, which is $50 \%$ to $55 \%$.

The authors point out that clinicians should be aware of the possibility of herpes zoster due to reactivation of vOka from zoster vaccine, but they can be reassured that the risk appears to be extremely low. Zoster vaccine offers an important opportunity for preventing herpes zoster and its often devastating complications.

Tseng HF, Schmid S, Harpaz R, et al. Herpes zoster caused by vaccine-strain varicella zoster virus in an immunocompetent recipient of zoster vaccine. Clin Infect Dis. 2014;58(8):11251128.

\section{RESTLESS LEG SYNDROME DUE TO THE COMBINATION OF VENLAFAXINE AND QUETIAPINE}

A 47-year-old female was admitted to the hospital with an exacerbation of her bipolar disorder. On admission, her medication history included valproate $1.5 \mathrm{~g}$ daily (valproate plasma level of $79 \mathrm{mg} / \mathrm{L}$; reference range, $55-100 \mathrm{mg} / \mathrm{L}$ ), topiramate $100 \mathrm{mg}$ daily, and venlafaxine $300 \mathrm{mg}$ daily. Quetiapine $150 \mathrm{mg}$ daily was initiated at bedtime for initial insomnia, agitation, and irritability and as an augmentation of antidepressant effect. Within 48 hours of receiving quetiapine, the patient began to complain of an uncomfortable tingling sensation deep inside her calves and jerks and an urge to move her legs, occurring exclusively at rest and strongly worsening at night. The unpleasant sensations were relieved partially when she stretched her legs and walked. Nevertheless, she experienced relief only as long as the activity continued, and symptoms reemerged after she stopped stretching and lying down again. Because these symptoms occurred only at night, the patient had problems initiating and maintaining sleep. The patient's venlafaxine was tapered, and this resulted in the elimination of her restless leg syndrome (RLS) within 48 hours. The patient was evaluated for possible secondary causes of RLS as well as family history, and all assessments were negative. The patient was discharged with the following medications; valproate $1.5 \mathrm{~g}$ daily, topiramate $100 \mathrm{mg}$ daily, and quetiapine $150 \mathrm{mg}$ daily.

After 6 months, the patient exhibited depressive symptoms and demonstrated psychomotor retardation, so her quetiapine was reduced to $100 \mathrm{mg}$ daily and venlafaxine $75 \mathrm{mg}$ daily was initiated. The patient's previously described RLS symptoms emerged after 48 hours. Her quetiapine was subsequently tapered and substituted with olanzapine $10 \mathrm{mg}$ daily. This intervention resulted in a prompt elimination of RLS symptoms. The patient has remained stable for 
the last 4 months, and her doses of valproate, topiramate, and venlafaxine have remained unchanged.

The authors stated that the patient fulfilled the diagnostic criteria for RLS, however the patient did not consent to polysomnography because of the short duration of RLS. Thus an association with periodic limb movement disorder could not be tested. The close temporal relationship between the concomitant administration of low doses of quetiapine and venlafaxine and the emergence of RLS may indicate a causative role of this combination. Neither quetiapine alone nor venlafaxine alone was associated with RLS in this patient.

The authors theorize that the mechanism by which antidepressants and antipsychotics might cause RLS remains unclear. Dopaminergic dysfunction of the subcortical dopamine pathways is considered to be a central pathophysiological mechanism in the development of RLS. Quetiapine has a moderate and more transient D2 dopamine receptor occupancy and a greater $5 \mathrm{HT}_{2 \mathrm{~A}}$ antagonism, which leads to an increase in synaptic availability of serotonin. The authors also speculate that RLS might have emerged under higher doses of quetiapine alone, but their patient never received them. Venlafaxine may enhance a serotonin-mediated inhibition of dopaminergic neurotransmission, contributing to dopaminergic hypoactivity.

The authors note that their patient did not manifest RLS with venlafaxine $300 \mathrm{mg}$ daily, although there was not any coadministration of quetiapine. She developed RLS only under the combined action of lower doses of venlafaxine and quetiapine, possibly because of the even greater dopaminergic hypoactivity induced by both drugs. The authors warn that clinicians should not overlook the possibility that RLS might be associated with the quetiapine-venlafaxine combination. If the drugs are given in combination, they suggest that the removal of one of the drugs might be beneficial in the successful treatment of RLS.

Michopoulos I, Ferentinos P, Oulis P, et al. Restless leg syndrome associated with the combined use of quetiapine and venlafaxine. J Clin Psychopharm. 2014;34(1):159-160.

\section{SSRIS AND THE RISK OF UPPER GASTROINTESTINAL BLEEDING}

The authors conducted an observational study evaluating the use of antidepressants between the years 1998 and 2009 in the Taiwan National Health Insurance Research Database (NHIRD). The researchers focused on patients with a psychiatric diagnosis who were newly diagnosed with upper gastrointestinal
(GI) bleeding and required hospitalization. A total of 187,117 patients were evaluated, and 5,377 patients met the study criteria and were included in the analysis. The authors examined the risk of upper GI bleeding during 3 time periods; 7 days after initiation, 14 days after initiation, and 28 days after initiation of an antidepressant. Antidepressants included in the analysis were selective serotonin reuptake inhibitors (SSRIs), monoamine oxidase (MAO) inhibitors, tricyclic antidepressants, serotonin norepinephrine reuptake inhibitors (SNRIs), and other agents including trazodone, mirtazapine, and bupropion. The SSRIs included in the analysis included fluoxetine, citalopram, paroxetine, sertraline, fluvoxamine, and escitalopram.

The researchers found that SSRIs were associated with an elevated risk of upper GI bleeding in all 3 time windows $(7,14$, and 28 days). The risk after 14 days of exposure was higher than that for the 7 and 28-day windows. Multiple mechanisms may be involved in this association. Within 14 days of use, SSRIs can significantly reduce intraplatelet serotonin concentration and impair platelet plug formation. SSRIs can also directly induce mucosal damage in the GI tract and stimulate gastric acid secretion that may potentiate the risk for upper GI bleeding.

Among the various SSRIs, fluoxetine and sertraline were most associated with an elevated risk of upper GI bleeding. Patients with preexisting GI bleeding were predisposed to bleeding after the initiation of an SSRI. Although other SSRIs, such as citalopram, paroxetine, and escitalopram, were associated with an elevated bleeding risk, the relationship fell short of statistical significance. The study may have been underpowered to detect an increased risk with the individual agents. Short-term exposure to tricyclic antidepressants, SNRIs, MAO inhibitors, and other antidepressants was not associated with an elevated risk of upper GI bleeding. The authors indicate that this finding suggests that these drugs might serve as alternatives to SSRIs in psychiatric patients with a history of GI bleeding or peptic ulcer disease.

The researchers also evaluated the possible effects of patients receiving nonsteroidal anti-inflammatory drugs (NSAIDs) or aspirin with an SSRI. An elevated risk of upper GI bleeding was observed in patients who were taking NSAIDS, SSRIs, or aspirin alone. For patients taking SSRIs with aspirin, the risk of upper GI bleeding was increased slightly more than SSRI monotherapy. The greatest upper GI bleeding risk was observed with the combination of SSRIs and NSAIDs. The researchers also found that male but not female SSRI users had an elevated risk for 
upper GI bleeding. Previous studies have also found a lower risk in female SSRI patients. There are several possible explanations for a lower risk in female patients. First, females have higher serotonin levels than males because estradiol can stimulate serotonin uptake by platelets. Second, estrogen can stimulate platelet aggregation, resulting in higher platelet aggregation activity in female patients. Last, males are at increased risk for peptic ulcer disease, and the gastric environment is more acidic in male patients.

The authors point out that their study demonstrated that short-term SSRI exposure (as little as 7 days) increased the risk of upper GI bleeding. In spite of the fact that SSRIs are widely used, they are associated with significant side effects. For example, there are reports of suicidality, birth defects, neonatal withdrawal syndrome, sexual dysfunction, hyponatremia, weight gain, insomnia, and GI bleeding with longterm use. This calls into question the extensive use of SSRIs, because there are evidence-proven alternative interventions available. The authors suggest that clinicians make treatment decisions on an individualized basis when prescribing SSRIs by balancing the potential side effects and treatment responses. Close monitoring of signs of GI bleeding may be warranted soon after initiating SSRI treatment.

Wang YP, Chen YT, Tsai CF, et al. Short-term use of serotonin reuptake inhibitors and risk of upper gastrointestinal bleeding. Am J Psych. 2014;171(1):54-61.

\section{MINOCYCLINE-INDUCED CUTANEOUS POLYARTERITIS NODOSA}

An 18-year-old female complained to her pediatrician of a 4-week history of a solitary painful bullous skin lesion over the lower right anterior tibia. The patient also complained of generalized myalgia, polyarthralgia, joint stiffness, and episodic ankle swelling. She stated that the lesion first appeared as a skin-colored papule and a red halo developed around the papule within a week. The patient's medical history included asthma and acne. Her medication history included minocycline hydrochloride for the last 25 months for acne, ranitidine, an oral contraceptive pill, and an iron supplement. She reported no family history of her current condition. The patient was treated with prednisone $10 \mathrm{mg}$ daily.

Seven weeks later, the initial skin lesion had evolved into an ulcer with a red, raised border. The patient had also developed new eruptions on both legs on the anterior tibia. The new eruption was consistent with livedo reticularis. Livedo reticularis is a netlike pattern of reddish-blue skin discoloration linked to swollen blood vessels. Based on these new findings, the patient underwent a battery of tests that included an elevated antinuclear antibody, elevated sedimentation rate, an elevated C-reactive protein, and an elevated serum IgG level. The following tests were performed but were normal or negative: complete blood count, urinalysis, liver profile, Lyme disease, parvovirus B19, Monospot test, uric acid, rheumatic factor, antistreptolysin antibody, anti-Scl70, antihepatitis $\mathrm{C}$ virus, hepatitis $\mathrm{B}$ surface antigen, hepatitis B core antibody, cytomegalovirus antibody, and human T-lymphotropic virus 1 and 2 antibodies. The skin lesion was cultured, but the results were negative. A further workup for systemic lupus erythematosus, including anti-Sjögren syndrome antigen $\mathrm{A}$, anti-Sjögren syndrome B, antiribonucleoprotein, anti-Sm, and anti-double-stranded DNA antibodies, was negative. Based on these findings and histologic results, the patient was diagnosed with cutaneous polyarteritis nodosa (CPN) due to minocycline.

$\mathrm{CPN}$ is a necrotizing vasculitis of small and mediumsized arteries within the skin, without involvement of the internal organs. Diagnostic guidelines proposed by Culver et al for minocycline-induced CPN include 7 criteria; patients should meet at least 6 for diagnosis. The 7 criteria are (1) minocycline use for more than 12 months; (2) skin manifestations, including livedo reticularis and/or subcutaneous nodules; (3) arthritis and/or myalgia and/or neuropathy in the distribution of the rash; (4) lack of systemic organ involvement; (5) skin biopsy sample showing necrotizing vasculitis of small and/or medium-sized vessels; (6) evidence of perinuclear antineutrophilic cytoplasmic antibodies; and (7) improvement after discontinuation of minocycline.

CPN is usually self-limiting once minocycline is discontinued. However the syndrome will recur if minocycline is restarted, therefore minocycline is contraindicated after CPN has occurred. This patient was treated with intermittent courses of prednisone for symptom management, and minocycline was discontinued. The patient had complete resolution of her symptoms, and her laboratory results returned to normal. The patient continues to be asymptomatic 18 months after discontinuation of minocycline. The authors recommend that clinicians be aware of this serious but reversible adverse effect of minocycline. Early recognition and discontinuation result in a favorable outcome with complete resolution of lesions.

Culver B, Itkin A, Pischel K, et al. Case report and review of minocycline-induced cutaneous polyarteritis nodosa. Arthritis Rheum. 2005;53(3):468-470.

Odhav A, Odhav C, Dayal N. Rare adverse effect of treatment with minocycline. JAMA Peds. 2014;168(3):287-288. 